\title{
Opération win-win pour le corps médical et le personnel infirmier
}

\author{
Isabelle Praplana, Esther Kraft ${ }^{b}$ \\ ${ }^{a}$ Cheffe de projet qualité et sécurité des patients, $\mathrm{H}+$ Les Hôpitaux de Suisse; ${ }^{b}$ Cheffe de la division Données, démographie et qualité (DDQ) de la FMH
}

Les Peer Reviews interprofessionnels ouvrent de nouvelles perspectives aussi bien aux membres du corps médical qu'au personnel infirmier en leur permettant d'élargir leur horizon. Tant le Prof. Jean-Blaise Wasserfallen du CHUV que la Prof. Rebecca Spirig de l'USZ ne trouvent que des avantages à la procédure interprofessionnelle. Ils se réjouissent que la Suisse fasse œuvre de pionnier.

L'examen par les pairs (Peer Review) est une procédure volontaire qui a fait ses preuves. Il consiste à analyser des cas a posteriori avec le concours de spécialistes externes et - le cas échéant - à élaborer en commun des mesures d'amélioration de la qualité. L'objectif est de susciter un processus interne d'amélioration continue et d'instaurer une culture ouverte de l'erreur et de la sécurité parmi les collaborateurs des hôpitaux et des cliniques. Créée il y a huit ans, l'Initiative pour une

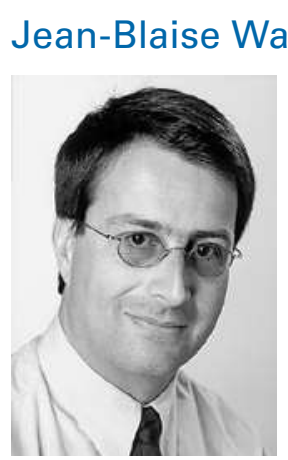

\section{erfallen}

Le Prof. Jean-Blaise Wasserfallen est directeur médical du Centre hospitalier universitaire vaudois (CHUV) à Lausanne, il siège au comité de I'IQM et dispose d'une expérience de plusieurs années dans les questions de sécurité des patients dont il fait bénéficier les Peer Reviews IQM. En sa qualité de directeur médical du CHUV, il a accompagné l'un des quatre projets pilotes et a fourni des indications décisives pour I'amélioration des Peer Reviews interprofessionnels. Selon lui, l'idéal serait qu'indépendamment d'un Peer Review IQM, les services et les groupes professionnels s'assoient spontanément autour d'une table pour analyser un cas spécifique et en tirer les enseignements.

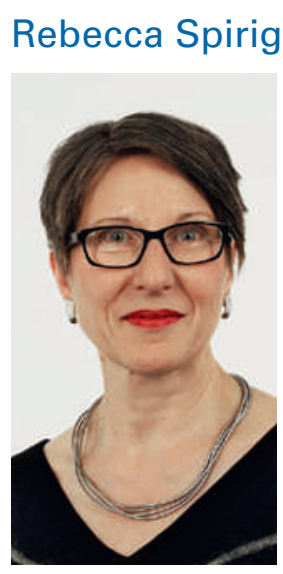

La Prof. Rebecca Spirig est directrice des soins infirmiers et responsable du service médico-technique et médico-thérapeutique (MTTB) de I'Hôpital universitaire de Zurich. Depuis 2016, elle représente les soins infirmiers au comité de I'IQM. En qualité de directrice des soins, elle a également une certaine expérience des Peer Reviews IOM. Elle exerce notamment un rôle important dans la recherche de nouveaux pairs infirmiers. Rebecca Spirig estime que les Peer Reviews interprofessionnels fonctionnent très bien, même si certains pairs infirmiers étaient sceptiques au départ. Pour la future collaboration entre les médecins et les infirmiers/infirmières dans le cadre des Peer Reviews, elle souhaite que les deux groupes professionnels soient sur pied d'égalité et fassent chacun preuve de compréhension mutuelle. Elle estime qu'ils pourront ainsi communiquer sans difficultés. médecine de qualité (Initiative Qualitätsmedizin e.V.) a développé une procédure de Peer Review reposant sur les données de routine, autrement dit sur les données de facturation. Il s'agit, pour les médecins-chefs, d'analyser la qualité des traitements en étudiant des dossiers de patients.

L'amélioration continue de la qualité des traitements médicaux est l'une des principales priorités des hôpitaux et des cliniques suisses. C'est pourquoi les institutions sont de plus en plus nombreuses à adhérer à l'IQM, à faire analyser des indicateurs sur la base de données de routine (par ex. les taux de mortalité) et à comparer leurs résultats à l'interne avec d'autres établissements membres de l'IQM.

\section{La Suisse pionnière}

Il y a deux ans, la Fédération des médecins suisses (FMH) et l'Association suisse des directrices et des directeurs des services infirmiers Swiss Nurse Leaders (anciennement ASDSI) ainsi que $\mathrm{H}+$ ont fondé l'Alliance Peer Review $\mathrm{CH}$ et porté sur les fonts baptismaux un projet de développement des Peer Reviews interprofessionnels. Il s'agissait d'examiner si et dans quelle mesure cette approche impliquant des pairs médecins et des pairs infirmiers était réalisable. Ont donc été testés d'une part la collaboration entre des responsables / experts des soins infirmiers et des médecins cadres, et d'autre part le fonctionnement de la procédure en Suisse romande. En 2014 et 2015, quatre soignants suisses ont suivi, avec des médecins-chefs, la formation continue de pair correspondant au curriculum de la Chambre médicale allemande. La documentation de l'IQM relative aux Peer Reviews a en outre été orientée sur les deux groupes professionnels et traduite en français. En 2015, des expériences pilotes ont été organisées 
Correspondance:

H+ Die Spitäler der Schweiz • H+ Les Hôpitaux de Suisse •

$\mathrm{H}+\mathrm{Gli}$ Ospedali Svizzeri

Secrétariat central

Lorrainestrasse $4 \mathrm{~A}$

CH-3013 Berne

isabelle.praplan[at]hplus.ch

Tél. 031335112

Contact FMH:

FMH Fédération des

médecins suisses

Division DDQ

Elfenstrasse 18 /

case postale 300

CH-3000 Berne 15

Esther.kraft[at]fmh.ch

Tél. 0313591111 dans quatre hôpitaux - l'Hôpital cantonal d'Argovie, l'Inselgruppe, le CHUV et les HUG. Les feedbacks ont été très positifs. Le projet ayant été couronné de succès, tous les Peer Reviews IQM se déroulent de manière interprofessionnelle en Suisse depuis 2016. Compte tenu des bonnes expériences réalisées en Suisse, l'IQM a repris la balle au bond et teste à son tour l'approche interprofessionnelle en Allemagne dans le cadre de projets pilotes.

\section{Plus-value pour les médecins}

Quels sont les avantages de la procédure interprofessionnelle pour les médecins? La réponse du Prof. JeanBlaise Wasserfallen, directeur médical du Centre hospitalier universitaire vaudois (CHUV) à Lausanne, est claire: «Ce ne sont pas seulement des équipes interdisciplinaires qui contribuent à l'ensemble de la procédure de traitement, mais aussi les représentants de diverses professions, tels que les médecins ou les membres du personnel infirmier. Dans un examen par les pairs, la perspective des différents groupes professionnels est donc impérative, en vue d'une amélioration continue.» Jean-Blaise Wasserfallen ajoute que le personnel infirmier joue un rôle important dans le processus de traitement, car c'est lui qui accompagne en permanence le patient et qui dispose d'une vue plus complète de son traitement et de ses besoins. Les Peer

\section{Alliance Peer Review}

L'Alliance Peer Review CH a été fondée en 2014 par la Fédération des médecins suisses (FMH) et I'Association suisse des directrices et des directeurs des services infirmiers Swiss Nurse Leaders (anciennement ASDSI) ainsi que par $\mathrm{H}+$. Sa mission consiste à développer des Peer Reviews interprofessionnels et uniformes au niveau suisse, sur la base de la procédure initiée en Allemagne par I'IOM. L'alliance s'est chargée de la conception des quatre projets pilotes qui se sont déroulés en automne 2015. Compte tenu des retours d'expériences positifs de la part des hôpitaux pilotes, I'Alliance Peer Review $\mathrm{CH}$ recommande aux institutions d'adhérer à I'IOM et préconise de réaliser systématiquement les Peer Reviews IQM sur une base interprofessionnelle dès 2016.

Reviews doivent donc aussi tenir compte de cet aspect. La directrice des soins infirmiers et responsable du service médico-technique et médico-thérapeutique (MTTB) de l'Hôpital universitaire de Zurich, la Prof. Rebecca Spirig, partage l'opinion du directeur médical du CHUV. Mais elle avance un avantage supplémentaire: «Les deux groupes professionnels ont la possibilité, lors de l'analyse du cas, d'apporter leur vision des choses, ce qui permet de brosser un tableau complet du traitement.» Elle insiste sur le fait que les groupes professionnels peuvent ainsi apprendre entre eux, mais aussi les uns des autres - autant dire une opération win-win. 